\title{
Fluid dynamics near the QCD critical point
}

\author{
Marcus Bleicher* and Christoph Herold \\ Institut für Theoretische Physik, Goethe-Universität, Max-von-Laue-Str. 1, 60438 Frankfurt am \\ Main, Germany \\ Frankfurt Institute for Advanced Studies (FIAS), Ruth-Moufang-Str. 1, 60438 Frankfurt am \\ Main, Germany \\ E-mail: bleichereth.physik.uni-frankfurt.de, \\ heroldeth.physik.uni-frankfurt.de
}

We present a fully dynamical model to study the chiral and deconfinement transition of QCD simultaneously. The quark degrees of freedom constitute a heat bath in local equilibrium for both order parameters, the sigma field and a dynamical Polyakov loop. The nonequilibrium evolution of these fields is described by Langevin equations including dissipation and noise. In several quench scenarios we are able to observe a delay in the relaxation times near the transition temperature for a critical point as well as a first-order phase transition scenario. During the hydrodynamical expansion of a hot quark fluid we find a strong enhancement of thermal fluctuations at the first-order transition compared to a scenario with a critical point.

Xth Quark Confinement and the Hadron Spectrum,

October 8-12, 2012

TUM Campus Garching, Munich, Germany

*Speaker. 


\section{Introduction}

The QCD phase diagram and the location of a possible critical point $(\mathrm{CP})$ are subject to intense theoretical and experimental research. Lattice QCD calculations support a crossover at vanishing baryon densities [1], while model studies indicate the existence of a first-order phase transition at high baryon densities ending at a CP [2]. In [3] it was shown how to detect the CP in heavy ion collisions by searching for divergences in event-by-event fluctuations of transverse momentum or particle multiplicity, an ansatz that has recently been refined to higher order cumulants $[4,5]$. However, it is expected that finite size and time effects and possibly initial state fluctuations will crucially weaken the expected signals $[6,7]$. On the other hand, the nonequilibrium evolution during a heavy-ion collision will enhance effects at the first-order phase transition, where spinodal instabilities may produce domain formation and clustering in energy and baryon density [8-10]. Hadronization of these clusters will lead to large non-statistical fluctuations in the hadron rapidity density within single events, providing an important observable signal for upcoming experiments at FAIR and NICA [11].

A successful dynamical model to study effects at the QCD phase transition in nonequilibrium has to go beyond usual hydrodynamics that includes the phase transition only in the equation of state [12]. A novel approach that includes the dynamics of the order parameters explicitly is given by chiral fluid dynamics [13-17]. We recently extended this model with the Polyakov loop to consider both the chiral and the deconfinement transition $[10,18]$.

\section{Polyakov-chiral fluid dynamics (P $\chi$ FD)}

The basic idea of the model is to explicitly propagate the sigma field and an effective Polyakov loop as the order parameters of the chiral and deconfinement phase transition. A fluid dynamically expanding medium of quarks and antiquarks provides the locally thermalized background for these fields. This enables us to study relevant effects at the $\mathrm{CP}$ and first-order transition in a dynamical system of finite size.

We use the Polyakov loop extended quark meson model [19] with the Lagrangian

$$
\mathscr{L}=\bar{q}\left[i\left(\gamma^{\mu} \partial_{\mu}-i g_{\mathrm{s}} \gamma^{0} A_{0}\right)-g \sigma\right] q+\frac{1}{2}\left(\partial_{\mu} \sigma\right)^{2}-U(\sigma)-\mathscr{U}(\ell, \bar{\ell})
$$

where $q=(u, d)$ is the constituent quark field, $A_{0}$ the temporal component of the color gauge field, $\sigma$ the mesonic field and $\ell$ the Polyakov loop. The pion degrees of freedom are neglected throughout this work. The potential for the sigma field is the usual "Mexican hat"

$$
U(\sigma)=\frac{\lambda^{2}}{4}\left(\sigma^{2}-v^{2}\right)^{2}-h_{q} \sigma-U_{0},
$$

and the temperature dependent Polyakov loop potential is chosen in a polynomial form $[19,20]$ :

$$
\frac{\mathscr{U}}{T^{4}}(\ell, \bar{\ell})=-\frac{b_{2}(T)}{4}\left(|\ell|^{2}+|\bar{\ell}|^{2}\right)-\frac{b_{3}}{6}\left(\ell^{3}+\bar{\ell}^{3}\right)+\frac{b_{4}}{16}\left(|\ell|^{2}+|\bar{\ell}|^{2}\right)^{2} .
$$


Integrating out the quark degrees of freedom in the partition function $\mathscr{Z}$ gives us the effective potential:

$$
V_{\text {eff }}=-\frac{T}{V} \ln \mathscr{Z}=U+\mathscr{U}+\Omega_{q \bar{q}} .
$$

Here, the quark contribution $\Omega_{q \bar{q}}$ determines the local equilibrium pressure of the quark fluid. In mean-field approximation and at zero chemical potential it reads [19]:

$$
\Omega_{q \bar{q}}=-4 N_{f} T \int \frac{\mathrm{d}^{3} p}{(2 \pi)^{3}} \ln \left[1+3 \ell \mathrm{e}^{-\beta E}+3 \ell \mathrm{e}^{-2 \beta E}+\mathrm{e}^{-3 \beta E}\right] .
$$

We tune the strength of the transition by varying the quark-meson coupling $g$. This allows us to study first-order phase transitions and transitions through the $\mathrm{CP}$ at vanishing baryochemical potential. Figure 1 shows the effective potential for $g=4.7$ (first-order) and $g=3.52(\mathrm{CP})$ at the respective transition temperature (cf. Ref. [10]). Note that in general one has to choose $g$ such that the product $g \sigma$ resembles the constituent quark mass in vacuum, leading to a value of $g \sim 3.3$.

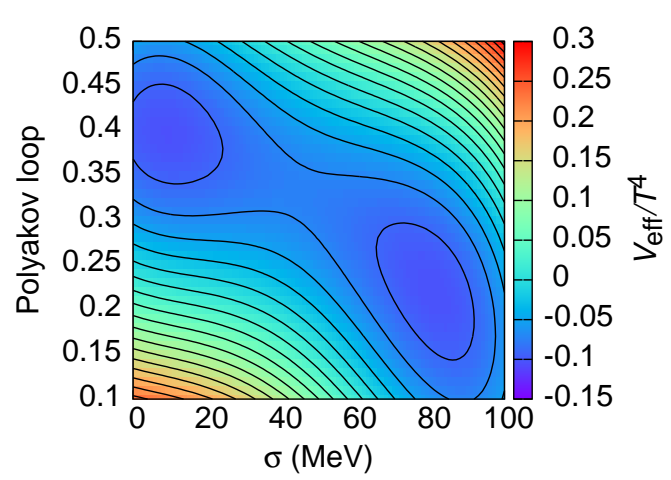

(a)

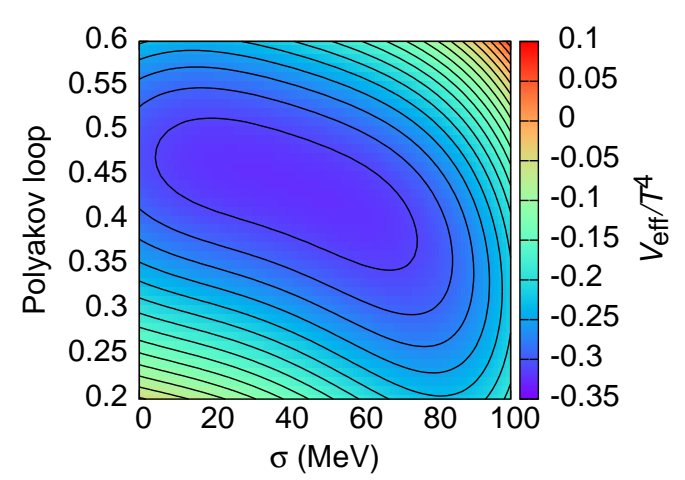

(b)

Figure 1: (a) Effective potential for $g=4.7$, corresponding to a first-order phase transition at $T_{c}=$ $172.9 \mathrm{MeV}$. (b) Effective potential for $g=3.52$, corresponding to a CP scenario at $T_{c}=180.5 \mathrm{MeV}$. Both figures are adopted from [10].

On can quantify this behavior by calculating the chiral and Polyakov loop susceptibilities $\chi_{\sigma \sigma}$ and $\chi_{\ell \ell}$. In Fig. 2 they are shown for three different couplings. We find divergent susceptibilities for $g=3.52$ indicating a chiral and deconfinement CP.

Within the two-particle irreducible effective action formalism we self-consistently derived the coupled dynamics for the sigma field and the quark heat bath [15]. We obtained a Langevin equation for the sigma field with temperature dependent damping $\eta_{\sigma}$ and stochastic noise term $\xi_{\sigma}$ that are connected via a dissipation fluctuation relation

$$
\begin{aligned}
\partial_{\mu} \partial^{\mu} \sigma+\eta_{\sigma}(T) \partial_{t} \sigma+\frac{\partial V_{\mathrm{eff}}}{\partial \sigma} & =\xi_{\sigma} \\
\left\langle\xi_{\sigma}(t, \vec{x}) \xi_{\sigma}\left(t^{\prime}, \vec{x}^{\prime}\right)\right\rangle & =\frac{1}{V} \delta\left(t-t^{\prime}\right) \delta\left(\vec{x}-\vec{x}^{\prime}\right) m_{\sigma} \eta_{\sigma} \operatorname{coth}\left(\frac{m_{\sigma}}{2 T}\right) .
\end{aligned}
$$




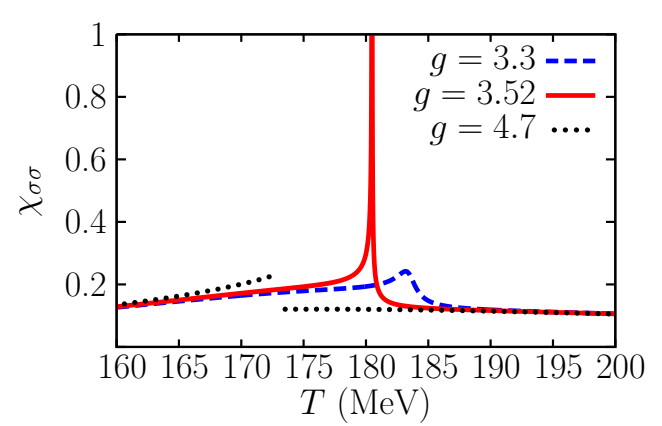

(a)

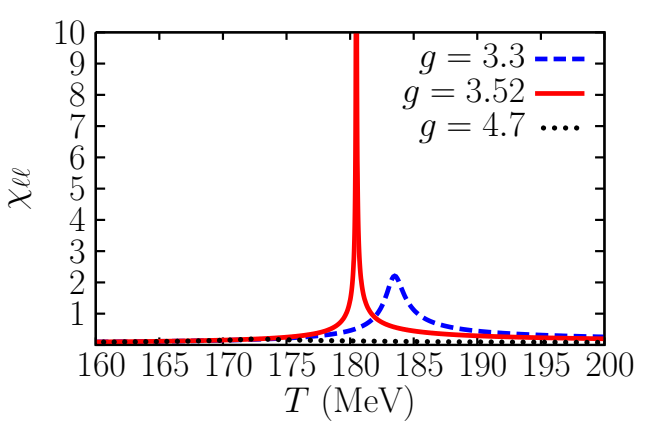

(b)

Figure 2: (a) Chiral susceptibility as a function of temperature for different coupling strengths. (b) Polyakov loop susceptibility as a function of temperature for different coupling strengths.

For the Polyakov loop field we deploy a relaxation equation which also contains stochastic noise:

$$
\begin{aligned}
\eta_{\ell} \partial_{t} \ell+\frac{\partial V_{\mathrm{eff}}}{\partial \ell} & =\xi_{\ell} \\
\left\langle\xi_{\ell}(t, \vec{x}) \xi_{\ell}\left(t^{\prime}, \vec{x}^{\prime}\right)\right\rangle T^{2} & =\frac{1}{V} \delta\left(t-t^{\prime}\right) \delta\left(\vec{x}-\vec{x}^{\prime}\right) 2 \eta_{\ell} T .
\end{aligned}
$$

Note here that the Polyakov loop is originally defined only in equilibrium and it is not a priori clear what the correct dynamics are [20]. This approach is therefore purely phenomenological. A similar ansatz with an additional kinetic term has been pursued in [21,22]. The damping coefficient $\eta_{\ell}$ is set to a value of $5 / \mathrm{fm}$. Results are sensitive to this choice only in the vicinity of the first-order transition temperature [10].

The quarks are propagated via the equations of ideal relativistic fluid dynamics:

$$
\partial_{\mu} T_{q}^{\mu v}=S_{\sigma}^{v}+S_{\ell}^{v}
$$

with source terms $S_{\sigma}^{v}$ and $S_{\ell}^{v}$ describing the energy transfer from the fields to the fluid via damping. The energy transfer due to stochastic fluctuations needs to be estimated numerically [10, 17].

\section{Numerical results}

\subsection{Equilibration in a box}

We study several temperature quenches in a cubic box with periodic boundary conditions. Both fields are initialized at some global $T_{i n i}>T_{c}$, with $T_{c}$ being the respective critical temperature. Then the temperature is quenched to a value $T<T_{c}$ and the energy density and pressure of the quark fluid are calculated. We let the coupled system evolve and relax. As pressure gradients are small within this setup, we expect the dynamics to be dominated by the fields. The solid red curves in Fig. 3 show the volume and event averaged sigma fiel $\overline{\langle\sigma\rangle}$ for equilibration near the transition point for both first-order and CP scenarios. At the first-order transition the significant delay in the relaxation time is caused by the large barrier separating the degenerate minima. Critical slowing down can be observed near the $\mathrm{CP}$, where the vanishing of $\eta_{\sigma}$ causes oscillations and prevents the field from relaxing to its equilibrium state. Similar effects occur in the Polyakov loop [10]. 


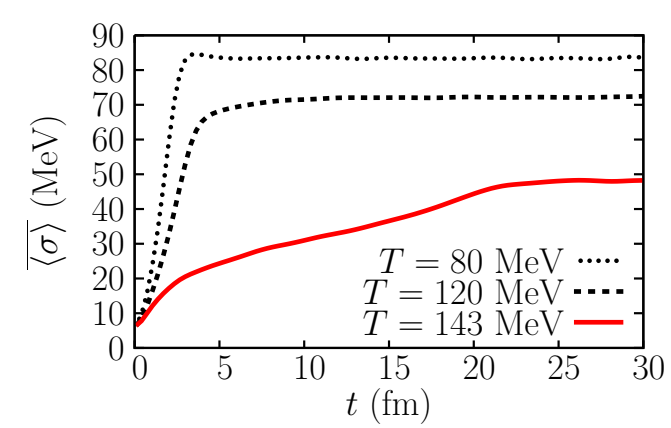

(a)

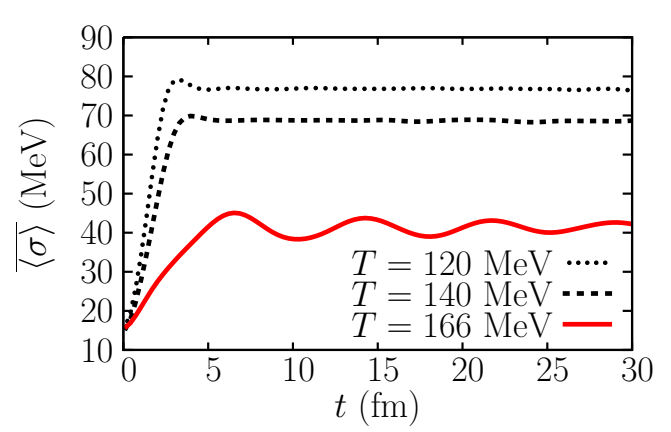

(b)

Figure 3: (a) Equilibration of the sigma field for several quench temperatures $T<T_{c}$ through the first-order transition. The barrier between the minima in the potential increases the relaxation time when the system relaxes near $T_{c}=172.9 \mathrm{MeV}$. We chose $T_{\text {ini }}=180 \mathrm{MeV}$. (b) Equilibration of the sigma field for several quench temperatures $T<T_{c}$ through the CP. Critical slowing down delays the dynamics and causes oscillations around the flat minimum when the system relaxes near $T_{c}=180.5 \mathrm{MeV}$. We chose $T_{\text {ini }}=186 \mathrm{MeV}$. Both figures are adopted from [10].

Another critical phenomenon can be observed by studying the intensity of field fluctuations. These are given for the sigma and Polyakov loop field as [10, 23]:

$$
\frac{\mathrm{d} N_{\sigma}}{\mathrm{d}^{3} k}=\frac{\omega_{k}^{2}\left|\delta \sigma_{k}\right|^{2}+\left|\partial_{t} \sigma_{k}\right|^{2}}{(2 \pi)^{3} 2 \omega_{k}}, \frac{\mathrm{d} N_{\ell}}{\mathrm{d}^{3} k}=T^{2} \frac{\omega_{k}^{2}\left|\delta \ell_{k}\right|^{2}+\left|\partial_{t} \ell_{k}\right|^{2}}{(2 \pi)^{3} 2 \omega_{k}}
$$

Here $\delta \sigma_{k}$ and $\partial_{t} \sigma_{k}$ are the $k$ th Fourier modes of $\delta \sigma=\sigma-\sigma_{\text {eq }}$ and $\partial_{t} \sigma$ and $\omega_{k}$ is the corresponding energy. We compare intensity histograms in the late stage of the evolution in the $\mathrm{CP}$ and first-order scenario in Fig. 4. For both order parameter fields we find a strong enhancement of long-wavelength modes at the $\mathrm{CP}$ compared to an equilibration near the first-order transition point.

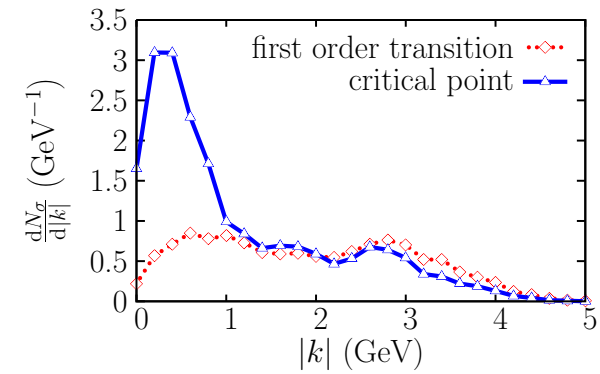

(a)

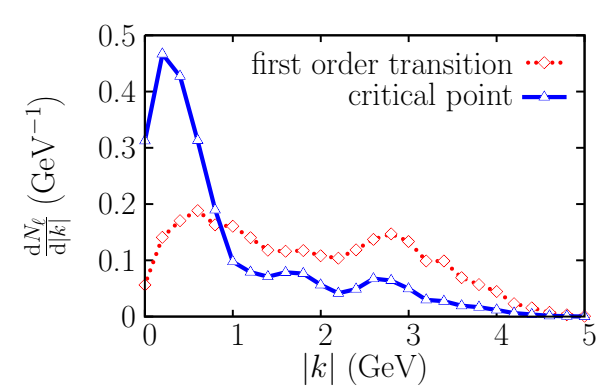

(b)

Figure 4: (a) Intensity of sigma fluctuations after equilibration at $t=24 \mathrm{fm}$. In the CP scenario we find an enhancement of the soft modes. (b) Intensity of Polyakov loop fluctuations after equilibration at $t=24 \mathrm{fm}$. In the CP scenario we find an enhancement of the soft modes. Both figures are adopted from [10].

\subsection{Fluid dynamic expansion}

To explore the influence of the expansion on the dynamics of the fields, an ellipsoidal region with a temperature $T=200 \mathrm{MeV}$, above both transition temperatures, is provided as initial state of a fluid dynamic expansion. This is to resemble the situation after the collision of two heavy nuclei. Fields and fluid are again set to their respective equilibrium values and the system evolves 
according to full (3+1)-dimensional fluid dynamics. During the expansion we observe supercooling and reheating in the first-order transition scenario. This supercooling causes an enhancement of nonequilibrium fluctuations $\langle\Delta \sigma\rangle=\sqrt{\left\langle\left(\sigma-\sigma_{\mathrm{eq}}\right)^{2}\right\rangle}$ and $\langle\Delta \ell\rangle=\sqrt{\left\langle\left(\ell-\ell_{\mathrm{eq}}\right)^{2}\right\rangle}$ in both order parameters at the first-order phase transition, see Fig. 5. The second bump in the fluctuation strength near $t=6 \mathrm{fm}$ arises when parts of the system cross the transition temperature a second time after reheating.

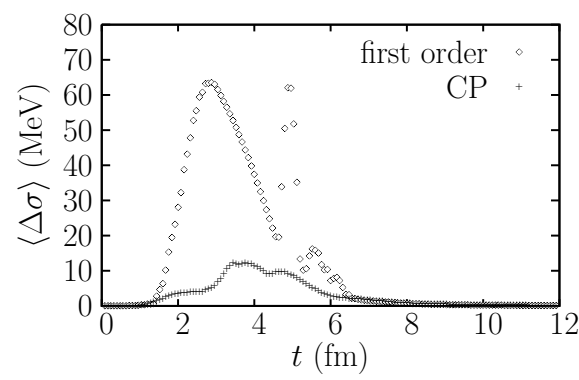

(a)

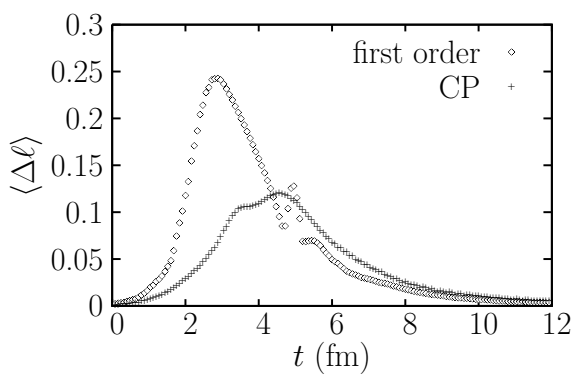

(b)

Figure 5: Nonequilibrium fluctuations of the sigma field (a) and Polyakov loop (b) are enhanced at the first-order transition compared to the CP scenario.

\subsection{Domain formation at the first-order phase transition}

We now focus on the evolution of a single event to learn more about the transition processes. To achieve this we introduce spatial correlations for the stochastic noise fields over volumes $1 / \mathrm{m}_{\sigma}^{3}$ and $1 / m_{\ell}^{3}$ to obtain a more physical behavior of these fluctuations.

We show a slice in the transversal $z=0$ plane for the sigma field, Polyakov loop and energy density in Figs. 6, 7 and 8, each for early, intermediate and late times in the evolution. We see in the order parameters domains of the high- and low-temperature phases coexisting during the transition process. This phenomenon is typical for the first-order phase transition and does not occur in evolutions through the CP. It can be best observed in the sigma field, but also the Polyakov loop exhibits a bumpy structure during its evolution. This structure then translates to the energy density, leading to a significant amount of inhomogeneity and clumping.

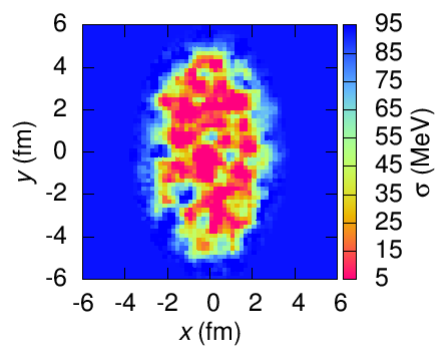

(a)

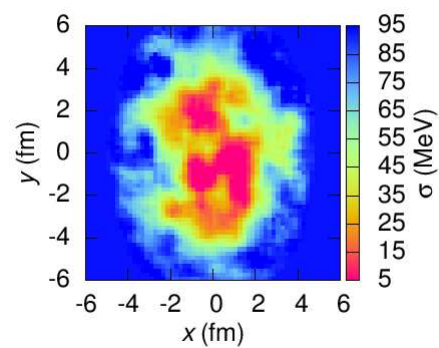

(b)

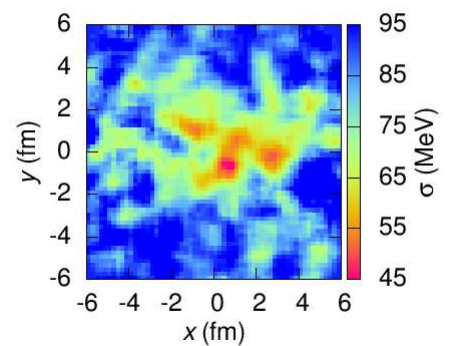

(c)

Figure 6: Sigma field in the $z=0$ plane for $t=1 \mathrm{fm}$ (a), $t=4 \mathrm{fm}$ (b), and $t=7 \mathrm{fm}$ (c) during a first-order phase transition. Fig. (b) adopted from [10]. 


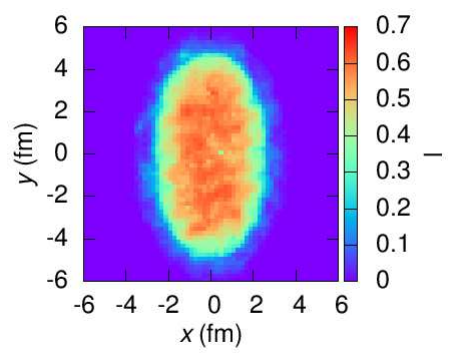

(a)

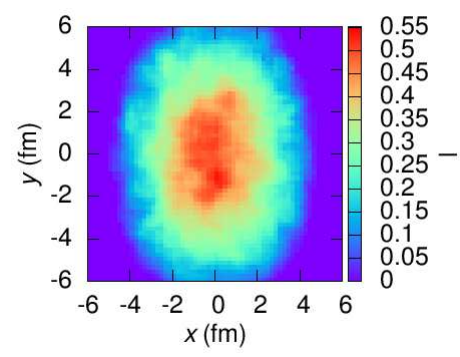

(b)

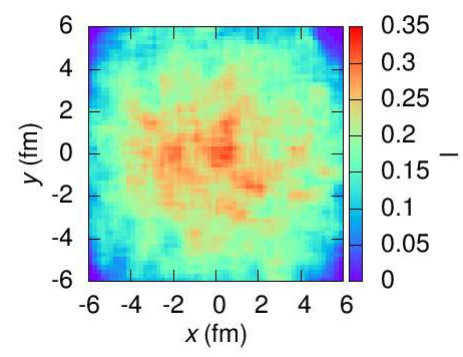

(c)

Figure 7: Polyakov loop field in the $z=0$ plane for $t=1 \mathrm{fm} \mathrm{(a),} t=4 \mathrm{fm}(\mathrm{b})$, and $t=7 \mathrm{fm}$ (c) during a first-order phase transition. Fig. (b) adopted from [10].

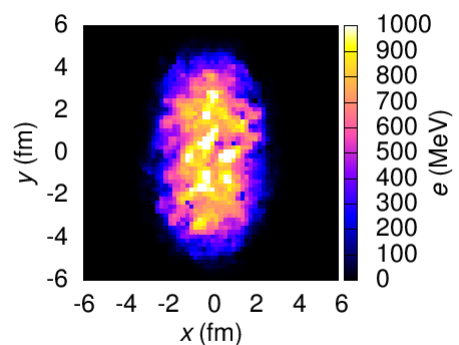

(a)

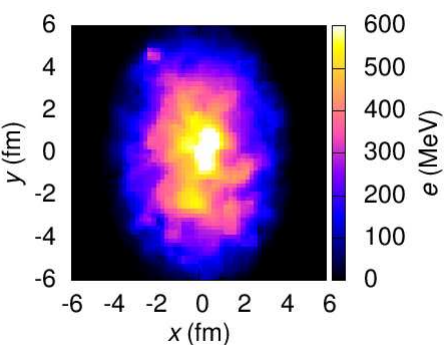

(b)

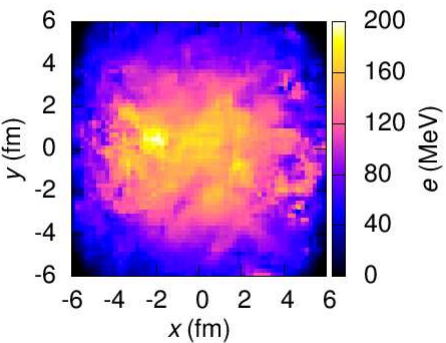

(c)

Figure 8: Energy density in the $z=0$ plane for $t=1 \mathrm{fm}$ (a), $t=4 \mathrm{fm}$ (b), and $t=7 \mathrm{fm}$ (c) during a first-order phase transition. Fig. (b) adopted from [10].

We expect this effect to become even stronger when we go to systems at finite baryon density. This would then provide an important experimental signal for the QCD phase transition, e. g. in non-monotonic multiplicity fluctuations of hadrons.

\section{Conclusions}

We presented the extension of nonequilibrium chiral fluid dynamics with a Polyakov loop to include effects of the deconfinement phase transition of QCD. We were able to observe typical critical phenomena like critical slowing down and the enhancement of soft modes for systems equilibrating near the CP. For an expanding system cooling through the first-order phase transition we found evidence for the formation of a supercooled phase leading to subsequent reheating of the fluid. As a result, large nonequilibrium fluctuations evolve. For single events, we find significant difference in the evolution of fields and fluid between the $\mathrm{CP}$ and the first-order scenario. The latter one proceeds through the formation of domains in the order parameter fields leading to irregularities in the energy density. As a next step we investigate this effect for systems at finite chemical potential to provide relevant signals of the QCD phase transition for upcoming experiments at FAIR. 


\section{Acknowledgments}

This work was supported by GSI and the Hessian LOEWE initiative Helmholtz International Center for FAIR.

\section{References}

[1] Y. Aoki, G. Endrodi, Z. Fodor, S. D. Katz and K. K. Szabo, Nature 443 (2006) 675-678.

[2] O. Scavenius, A. Mocsy, I. N. Mishustin and D. H. Rischke, Phys. Rev. C 64 (2001) 045202.

[3] M. A. Stephanov, K. Rajagopal and E. V. Shuryak, Phys. Rev. D 60 (1999) 114028.

[4] M. A. Stephanov, Phys. Rev. Lett. 102 (2009) 032301.

[5] F. Karsch and K. Redlich, Phys. Lett. B 695 (2011) 136.

[6] M. Bleicher et al., Nuc. Phys. A 638 (1998) 391c-394c.

[7] B. Berdnikov and K. Rajagopal, Phys. Rev. D 61 (2000) 105017.

[8] C. Sasaki, B. Friman and K. Redlich, Phys. Rev. D 77 (2008) 034024.

[9] J. Steinheimer and J. Randrup, Phys. Rev. Lett. 109 (2012) 212301.

[10] C. Herold, M. Nahrgang, I. Mishustin and M. Bleicher, Phys. Rev. C 87 (2013) 014907.

[11] I. N. Mishustin, Phys. Rev. Lett. 82 (1999) 4779.

[12] J. Steinheimer, M. Bleicher, H. Petersen, S. Schramm, H. Stocker and D. Zschiesche, Phys. Rev. C 77 (2008) 034901.

[13] I. N. Mishustin and O. Scavenius, Phys. Rev. Lett. 83 (1999) 3134.

[14] K. Paech, H. Stoecker and A. Dumitru, Phys. Rev. C 68 (2003) 044907.

[15] M. Nahrgang, S. Leupold, C. Herold and M. Bleicher, Phys. Rev. C 84 (2011) 024912.

[16] M. Nahrgang, S. Leupold and M. Bleicher, Phys. Lett. B 711 (2012) 109.

[17] M. Nahrgang, C. Herold, S. Leupold, I. Mishustin and M. Bleicher, arXiv:1105.1962 [nucl-th].

[18] C. Herold, M. Bleicher and M. Nahrgang, Acta Phys. Polon. Supp. 5 (2012) 529.

[19] B. -J. Schaefer, J. M. Pawlowski and J. Wambach, Phys. Rev. D 76 (2007) 074023.

[20] C. Ratti, M. A. Thaler and W. Weise, Phys. Rev. D 73 (2006) 014019.

[21] A. Dumitru and R. D. Pisarski, Phys. Lett. B 504 (2001) 282.

[22] A. Dumitru and R. D. Pisarski, Nucl. Phys. A 698 (2002) 444.

[23] A. Abada and M. C. Birse, Phys. Rev. D 55 (1997) 6887. 